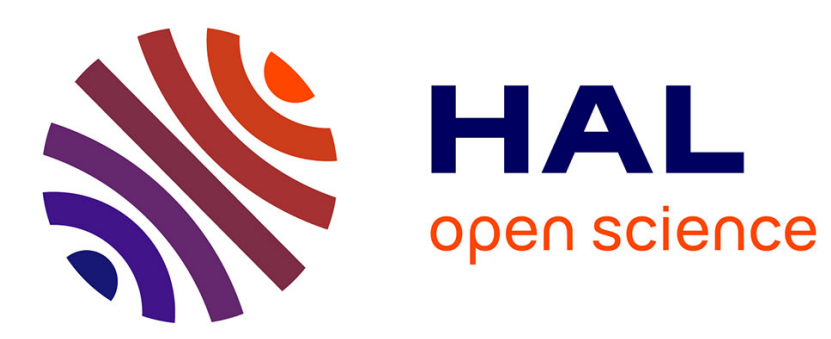

\title{
Complete Mechanical Regularization Applied to Digital Image and Volume Correlation
}

\author{
Arturo Mendoza, Jan Neggers, François Hild, Stéphane Roux
}

\section{To cite this version:}

Arturo Mendoza, Jan Neggers, François Hild, Stéphane Roux. Complete Mechanical Regularization Applied to Digital Image and Volume Correlation. Computer Methods in Applied Mechanics and Engineering, 2019, 355, pp.27-43. 10.1016/j.cma.2019.06.005 . hal-02148780

\section{HAL Id: hal-02148780 \\ https://hal.science/hal-02148780}

Submitted on 5 Jun 2019

HAL is a multi-disciplinary open access archive for the deposit and dissemination of scientific research documents, whether they are published or not. The documents may come from teaching and research institutions in France or abroad, or from public or private research centers.
L'archive ouverte pluridisciplinaire HAL, est destinée au dépôt et à la diffusion de documents scientifiques de niveau recherche, publiés ou non, émanant des établissements d'enseignement et de recherche français ou étrangers, des laboratoires publics ou privés. 


\title{
Complete Mechanical Regularization Applied to Digital Image and Volume Correlation
}

\author{
Arturo Mendoza ${ }^{\mathrm{a}, \mathrm{b}}$, Jan Neggers ${ }^{\mathrm{a}}$, François Hild ${ }^{\mathrm{a}}$, Stéphane Roux ${ }^{\mathrm{a}}$ \\ ${ }^{a}$ LMT (ENS Paris-Saclay/CNRS/Univ. Paris-Saclay), 94235 Cachan, France \\ ${ }^{b}$ Safran Tech, 78772 Magny les Hameaux, France
}

\begin{abstract}
This paper presents a new regularization scheme for Digital Image Correlation (DIC) and Digital Volume Correlation (DVC) techniques based on the equilibrium gap method with reference to a linear elastic behavior. This scheme constitutes a unique framework for performing the so-called mechanical regularization for any problem dimension. "Complete regularization" refers to the fact that a specific treatment of boundaries (surfaces) is introduced here on the same footing as the bulk, independently of the complexity of their shape. The proposed treatment distinguishes the roles that different boundaries (Neumann or Dirichlet) play in mechanical tests. Numerical cases on synthetic data and a real experimental test validate the robustness and accuracy of the method. The analyzed experiment shows that only the use of (complete) regularization ensures convergence. Even in the cases where such regularization is not employed but convergence is achieved, it is at much higher cost. These results reveal the benefit of regularization on the convergence rate of DVC.

Keywords: digital image correlation, digital volume correlation, mechanical regularization, equilibrium gap
\end{abstract}

\section{Introduction}

Digital Image Correlation (DIC) and Digital Volume Correlation (DVC) are popular techniques to measure displacement fields from image pairs in respectively $2 \mathrm{D}$ and $3 \mathrm{D}$ settings $[1,2]$. As a true three-dimensional technique, DVC

Preprint submitted to Computer Methods in Applied Mechanics and EngineeringJune 5, 2019 
measures the internal displacement fields from a pair of reconstructed volumes (3D images). These image registration techniques face a considerable challenge, namely, their ill-posedness [1,3,4]. The limited available information (i.e., intensity levels) leads to an unavoidable compromise between the measurement uncertainty and the spatial resolution $[5,6]$. Hence, small scale displacement resolutions are hardly accessible.

However, different approaches have been designed to overcome this limitation. For instance, the displacement field can be assumed continuous over the entire Region Of Interest (ROI). Thus, it can be decomposed over basis functions that fulfill this constraint. A convenient choice is offered by meshes used in the Finite Element (FE) method [7-9]. It is worth mentioning that meshless techniques have also been proposed in the context of DIC [10]. Since the solution is determined through the solution to a problem coupling all degrees of freedom, such techniques are referred to as global DIC and DVC [9, 11]. These approaches differ from their "local" counterparts [1, 2, 12, 13], which do not assume any continuity in the sought displacement fields.

One of the advantages of this approach is that it allows for a straightforward connection between experiments and simulations $[9,14]$. The same FE mesh used for measuring the displacement field, can also be used in numerical analyses. For example, the measurement of "real" boundary conditions using DVC can be used for guiding micromechanical simulations without the need for additional multiscale schemes [15].

Next, given that the studied images are discretized using pixels or voxels, quadrangle and cube elements are a natural choice. Yet, those elements have been shown to lead to "soft modes" with poor conditioning, thereby leading to specific diagonal patterns either when the small element size or the poor texture challenge noise sensitivity [16].

Alternatively, current meshing tools are capable of meshing objects with arbitrary complex boundaries using tetrahedral / triangular elements [17]. The use of such meshes for DVC opens many possibilities. For instance, the analysis would no longer be limited to ROIs with flat straight surfaces, or at the interior 
of the sample. Even if the sample has a complex shape, or simply because it has been slightly tilted during image acquisition, the ROI could conform to the actual boundary.

Additionally, regularization techniques [18] are employed to further circumvent the ill-posedness of the registration [3], or to limit ill-conditioning of incremental corrections, and therefore mitigate noise sensitivity [19, 20]. Often, some a priori information on the mechanical behavior of the studied material is available. Then, it is natural to seek a displacement field that best registers the images while also being mechanically admissible. That is the goal of the so-called mechanical regularization based on the equilibrium gap method [21]. This type of regularization can be seen as a specialized filter that only acts on spurious displacements if they are inconsistent with equilibrium.

This regularization constrains the displacement field to one that locally follows a linear elastic behavior. It allows displacements to be measured on meshes with elements of size comparable to the voxel size [22]. Elastic regularization has proven useful even when the actual behavior is more complex. For instance, the study of plastic flow was reported for a controlled crack propagation in aluminum alloy sheets [23]. The proper tuning of the elastic regularization helps enforcing the isochoric constraint, and freeing the direction of easy slip.

Even though this approach is valid independently of the type of mesh, it is not capable of applying the adequate regularization to each type of surface present in the analysis. In fact, the guiding principle is only valid for the bulk and free-surfaces of the studied sample. In reference [20], the authors proposed an approach that mimics the bulk, as if those surfaces had an elasticity of their own in addition to the bulk (as a kind of "surface tension"). However, the link between both models (bulk and surfaces) is poor (apart from using common nodes). Moreover, the technique is only applicable to $2 \mathrm{D}$ cases, and only admits regions of interest with straight boundaries.

This paper presents an extension of the mechanical regularization to a lowpass filtering of surface tractions. In this context, Saint-Venant's principle may be invoked, namely, harmonically modulated surface tractions applied to the 
surface of an elastic medium only affect a boundary layer whose thickness is equal to the wavelength [24]. Consequently, filtering out high frequency modes has a very limited effect, and remains in a confined region. Moreover, the resulting surface tractions over long wavelengths are to be preserved with no alteration. This is precisely what is achieved by the proposed regularization of Dirichlet surfaces. Furthermore, while the present application is 3D, the method can be easily applied to $2 \mathrm{D}$ configurations.

The implementation details are given in Section 2. Numerical tests are performed in Section 3, and an actual test case is presented in Section 4. These results will show the superiority of the proposed method in comparison with established DVC approaches.

\section{Regularization Method}

The present section addresses the methodology in general terms so as to be applicable to DVC (3D) and DIC (2D) analyses.

\subsection{Image Correlation}

The registration of the image in the reference configuration $f(\boldsymbol{x})$, and deformed configuration $g(\boldsymbol{x})$ is based on the brightness conservation assumption $[1,4]$

$$
f(\boldsymbol{x})=g(\boldsymbol{x}+\boldsymbol{u}(\boldsymbol{x})),
$$

where $\boldsymbol{u}(\boldsymbol{x})$ is the sought displacement field that minimizes the $L_{2}$ norm of the so-called "correlation residuals", $\eta(\boldsymbol{x})$,

$$
\eta(\boldsymbol{x})=g(\boldsymbol{x}+\boldsymbol{u}(\boldsymbol{x}))-f(\boldsymbol{x}),
$$

which is evaluated at each voxel (or pixel) location $\boldsymbol{x}$ of the entire ROI $\Omega$,

$$
\Phi_{c}=\sum_{\Omega} \eta(\boldsymbol{x})^{2} .
$$

The minimization of $\Phi_{c}$ is an ill-posed problem [3]. Thus, a weak formulation is chosen so as to decompose the displacement field $\boldsymbol{u}$ over a set of a priori chosen 
kinematics fields $\psi_{i}$, such as those used in the framework of the finite element (FE) method [25]

$$
\boldsymbol{u}(\boldsymbol{x})=\sum_{i} u_{i} \boldsymbol{\psi}_{i}(\boldsymbol{x})
$$

The registration problem then consists in minimizing $\Phi_{c}$ with respect to all the unknown amplitudes $u_{i}$. A Gauss-Newton scheme $[26,27]$ leads to the following linear system [4]

$$
\left[\boldsymbol{M}^{c}\right]\{\delta \boldsymbol{u}\}=\{\boldsymbol{b}\}
$$

in terms of the corrections $\{\delta \boldsymbol{u}\}$ to the sought degrees of freedom $u_{i}$ during the iterations, and

$$
\begin{aligned}
M_{i j}^{c} & =\sum_{\Omega}\left(\boldsymbol{\psi}_{i}(\boldsymbol{x}) \cdot \nabla f(\boldsymbol{x})\right)\left(\boldsymbol{\psi}_{j}(\boldsymbol{x}) \cdot \boldsymbol{\nabla} f(\boldsymbol{x})\right), \\
b_{i} & =\sum_{\Omega}\left(\boldsymbol{\psi}_{i}(\boldsymbol{x}) \cdot \nabla f(\boldsymbol{x})\right) \eta(\boldsymbol{x}),
\end{aligned}
$$

where the vector $\{\boldsymbol{b}\}$ accounts for the current image residuals $\eta(\boldsymbol{x})$, and the Hes$\operatorname{sian}\left[\boldsymbol{M}^{c}\right]$ is built from the shape functions $\boldsymbol{\psi}_{i}$ and the image gradient $\boldsymbol{\nabla} f(\boldsymbol{x})$, thus computed only once.

\subsection{Mechanical Regularization}

Many regularization techniques are possible for this ill-posed problem [28]. For example, a classical "soft" Tikhonov regularization [18] can be used to ensure smoothness of the solution. Furthermore, it can be argued that the correlation procedure embeds a natural form of regularization, namely, the $\mathrm{FE}$ mesh. Therefore, using bigger element sized meshes also constitutes a "hard" regularization scheme. That is the philosophy behind the pyramidal multiscale approaches $[9,11]$. Here, the kinematic degrees of freedom are reduced so as to avoid local minima trapping in the intermediate stages of the algorithm. Since, at each scale, the converged field is used as an initialization for the finer scale, this multiscale driving provides both robustness and low measurement uncertainties. However, such a procedure is not easily implemented with an unstructured mesh decomposition unless the mesh is endowed with a hierarchical 
structure. An alternative soft regularization that does not interfere with the mesh structure is needed.

\subsubsection{Equilibrium Gap}

In the spirit of what was introduced earlier, an ideal regularization would be based on the actual mechanical behavior of the studied specimen. Yet, in order to provide a general approach that does not need to be tailored to each individual case, a "simplification" of the aforementioned behavior is in order. That is, to acknowledge that locally, unless a localization instability occurs, the sought displacement may often be well described by an elastic problem. This is the spirit of the proposed regularization based on the equilibrium gap, namely, the "distance" between the current solution and that which locally satisfies the equilibrium equation for linear elasticity $[19,20,22,23]$. It is worth noting that an alternative approach is based on the Laplacian operator [29, 30]. However,

such regularization is not rotationally invariant and hence risks to alter the true kinematics.

The material deformation is measured via the infinitesimal strain tensor, $\boldsymbol{\epsilon}$,

$$
\boldsymbol{\epsilon}=\frac{1}{2}\left(\nabla \boldsymbol{u}+\nabla \boldsymbol{u}^{\boldsymbol{\top}}\right),
$$

which, in conjunction with the elasticity tensor $\boldsymbol{C}$, provide the stress tensor $\boldsymbol{\sigma}$ through the constitutive equation

$$
\sigma=C: \epsilon
$$

The equilibrium equation then satisfies

$$
\nabla \cdot \sigma+f=0
$$

where $\boldsymbol{f}$ is the distribution of body forces. In the context of the FE method [25, 31 , the combination of the above three equations assumes a discretized form

$$
[\boldsymbol{K}]\{\boldsymbol{u}\}=\{\boldsymbol{f}\},
$$

where $[\boldsymbol{K}]$ is the stiffness matrix, $\{\boldsymbol{f}\}$ the vector of nodal forces, and $\{\boldsymbol{u}\}$ collects the nodal displacements associated with the discretized displacement field $\boldsymbol{u}$. 
At this point, it is useful to recall the concept of "Neumann" and "Dirichlet" degrees of freedom (DOFs). These terms (Neumann and Dirichlet) are usually employed to denote the DOFs for which externally applied traction or displacement, respectively, are known, and where conversely displacement or traction are to be computed.

It should be noted that under this FE discretization, the studied DOFs can be classified into different groups. In order to better introduce each of them, let us use the binary diagonal projection matrices $\boldsymbol{D}$. These square matrices are valued 1 for the DOFs that pertain to the corresponding group and valued 0 otherwise. Then, given the identity matrix $\boldsymbol{I}$ that accounts for all the DOFs in the mesh, they can be categorized into

$$
\boldsymbol{I}=\boldsymbol{D}_{\mathcal{B}}+\boldsymbol{D}_{\mathcal{S}}
$$

where $\boldsymbol{D}_{\mathcal{S}}$ represents all the DOFs of surface nodes (i.e., those defined on the boundaries of the domain), and $\boldsymbol{D}_{\mathcal{B}}$ all the remaining (bulk) DOFs. Additionally, the surface DOFs are subdivided into

$$
D_{\mathcal{S}}=D_{\mathcal{S}_{\mathcal{N}}}+D_{\mathcal{S}_{\mathcal{D}}}
$$

where $\boldsymbol{D}_{\mathcal{S}_{\mathcal{N}}}$ and $\boldsymbol{D}_{\mathcal{S}_{\mathcal{D}}}$ denote the DOFs belonging to the Neumann and Dirichlet boundaries, respectively. While the former correspond to the DOFs whose force is known, the latter correspond to those for which the force is unknown (but displacements are prescribed). In particular, the Dirichlet boundary DOFs are listed as

$$
\boldsymbol{D}_{\mathcal{S}_{\mathcal{D}}}=\sum_{i} \boldsymbol{D}_{\mathcal{S}_{i}},
$$

where $\boldsymbol{D}_{\mathcal{S}_{i}}$ is the projection matrix associated with the (Dirichlet) surface $\mathcal{S}_{i}$.

It is worth mentioning that for the sake of readability, the $2 \mathrm{D}$ surfaces of a 3D mesh (for DVC cases) and the 1D boundaries of a 2D mesh (for DIC analyses) are both being referred to as "surfaces." Moreover, these boundaries can take any shape and are not limited to straight lines (as 1D boundaries) or flat planes (as 2D surfaces). The corresponding projection matrices $\boldsymbol{D}_{\mathcal{S}_{i}}$ only require that the connectivity be obeyed. 


\subsubsection{Regularization Principle}

Mechanical regularization consists in minimizing the $L_{2}$ norm of the unbalanced nodal forces for all bulk and Neumann DOFs. For the latter it is assumed that they correspond to traction-free surfaces. This is written as

$$
\Phi_{m}(\{\boldsymbol{u}\})=\left(\{\boldsymbol{u}\}^{\boldsymbol{\top}}\left[\boldsymbol{K}_{m}\right]^{\boldsymbol{\top}}-\{\boldsymbol{f}\}^{\boldsymbol{\top}}\right)\left(\left[\boldsymbol{K}_{m}\right]\{\boldsymbol{u}\}-\{\boldsymbol{f}\}\right),
$$

where $\Phi_{m}$ corresponds to the equilibrium gap for the bulk and Neumann surfaces DOFs, as given by the partial stiffness matrix

$$
\left[\boldsymbol{K}_{m}\right]=\left(\left[\boldsymbol{D}_{\mathcal{B}}\right]+\left[\boldsymbol{D}_{\mathcal{S}_{\mathcal{N}}}\right]\right)[\boldsymbol{K}]
$$

However, the same argument cannot be made for the remaining DOFs (i.e., those belonging to the Dirichlet surfaces) because the external forces are un-

known. Since the nodal forces for these DOFs do not vanish, it is proposed to introduce a new penalization term that tends toward a common local orientation and similar magnitude. Hence, a penalty is introduced on the quadratic norm of the gradient along each surface $\mathcal{S}_{i}$ of each component of the normal traction. Rapid variations of the unbalanced forces along the Dirichlet surfaces are discouraged. However, in order to obtain a global force that balances the resultant forces, the long wavelength components (including torques as moments of externally applied forces) are preserved without (or with a very low) penalty. This point is important in order not to prevent reaching the proper solution because of a heuristic smoothness assumption, which does not intend to reflect reality. Under a continuous setting, this surface regularization cost function is written as

$$
\Phi_{\mathcal{S}_{i}}=\int_{\mathcal{S}_{i}}\|\nabla(\boldsymbol{\sigma} \cdot \boldsymbol{n})\|^{2} \mathrm{~d} x,
$$

where $\boldsymbol{n}$ is the outward surface normal.

The surface gradient operator with the proposed FE discretization is denoted by $[\boldsymbol{G}]$. The $L_{2}$ norm of the gradient of (surface) tractions involves

$$
[\boldsymbol{L}]=[\boldsymbol{G}]^{\boldsymbol{\top}}[\boldsymbol{G}]
$$


which can be recognized as the discrete Laplace-Beltrami operator acting on such surface. This Laplacian can be computed, in a variational formulation [32$34]$, as the product of two symmetric matrices

$$
[\boldsymbol{L}]=[\boldsymbol{A}][\boldsymbol{B}],
$$

where the mass matrix $A_{i j}=\left\langle\boldsymbol{\varphi}_{i}, \boldsymbol{\varphi}_{j}\right\rangle^{-1}$ and the sparse matrix $B_{i j}=\left\langle\boldsymbol{\nabla} \boldsymbol{\varphi}_{i}, \boldsymbol{\nabla} \boldsymbol{\varphi}_{j}\right\rangle$ are defined using the $L_{2}$-inner product $\langle$,$\rangle and the shape functions \boldsymbol{\varphi}_{i}$.

It is worth noting that the shape functions $\varphi(x)$ are not the same as those employed earlier (i.e., $\boldsymbol{\psi}(\boldsymbol{x})) ; \boldsymbol{\varphi}(\boldsymbol{x})$ are the "restriction" of $\boldsymbol{\psi}(\boldsymbol{x})$ on the boundary. While the latter ones provide the kinematic decomposition for the complete mesh, the former ones define the "skin" of the mesh and thus only exist on the mesh boundaries. As such, the "original" finite elements with shape functions $\boldsymbol{\psi}(\boldsymbol{x})$ will generate "boundary" finite elements with shape functions $\boldsymbol{\varphi}(\boldsymbol{x})$ by simple "decomposition." For example, from a (3D) tetrahedral element will originate four (2D) triangular elements, and a (2D) triangular element will lead to three (1D) line elements. This procedure defines the corresponding (Dirichlet) surfaces $\mathcal{S}_{i}$ and their associated projection matrices $\boldsymbol{D}_{\mathcal{S}_{i}}$.

With the proposed FE discretization, the surface regularization consists in adding the following cost function for each surface $\mathcal{S}_{i}$

$$
\begin{aligned}
\Phi_{\mathcal{S}_{i}}(\{\boldsymbol{u}\}) & =\left\|[\boldsymbol{G}]\left[\boldsymbol{K}_{\mathcal{S}_{i}}\right]\{\boldsymbol{u}\}\right\|^{2}, \\
& =\{\boldsymbol{u}\}^{\top}\left[\boldsymbol{K}_{\mathcal{S}_{i}}\right]^{\top}[\boldsymbol{L}]\left[\boldsymbol{K}_{\mathcal{S}_{i}}\right]\{\boldsymbol{u}\},
\end{aligned}
$$

where $\Phi_{\mathcal{S}_{i}}$ corresponds to the penalization for DOFs belonging to the (Dirichlet) surface $\mathcal{S}_{i}$, as given by the partial stiffness matrix

$$
\left[\boldsymbol{K}_{\mathcal{S}_{i}}\right]=\left[\boldsymbol{D}_{\mathcal{S}_{i}}\right][\boldsymbol{K}]
$$

Let us stress that the bulk regularization is physically founded, and quite generic; although setting the elastic properties is a point that deserves discussions. Conversely, along the surfaces $\mathcal{S}_{i}$, the above suggested regularization is heuristic and aims at producing smooth fields. It is to be emphasized that no extraneous elasticity or surface tension is introduced as surface tractions are 
computed from bulk elasticity. As such, the proposed approach is readily applicable without the need for additional constraints, such as the "boundary" elements being shell elements instead of the simpler linear ones being used. Moreover, filtering out high frequency modes of surface tractions only smooths out a boundary layer close to the regularized surface, and leaves low frequency resultant force/torque unaffected.

\subsection{Weighting Scheme}

The minimization of residuals from the registration $\Phi_{c}$, the equilibrium gap $\Phi_{m}$, and the "loaded" surfaces $\Phi_{\mathcal{S}_{i}}$ requires the introduction of a total functional $\Phi_{t}$, whose weights are discussed hereafter [20-22]. Let us define

$$
\Phi_{t}(\{\boldsymbol{u}\})=\frac{\omega_{c}}{\omega_{t}} \hat{\Phi}_{c}(\{\boldsymbol{u}\})+\frac{\omega_{m}}{\omega_{t}} \hat{\Phi}_{m}(\{\boldsymbol{u}\})+\sum_{i} \frac{\omega_{\mathcal{S}_{i}}}{\omega_{t}} \hat{\Phi}_{\mathcal{S}_{i}}(\{\boldsymbol{u}\})
$$

with

$$
\begin{gathered}
\hat{\Phi}_{c}(\{\boldsymbol{u}\})=\Phi_{c}(\{\boldsymbol{u}\}) / E_{c}, \\
\hat{\Phi}_{m}(\{\boldsymbol{u}\})=\Phi_{m}(\{\boldsymbol{u}\}) / E_{m}, \\
\hat{\Phi}_{\mathcal{S}_{i}}(\{\boldsymbol{u}\})=\Phi_{\mathcal{S}_{i}}(\{\boldsymbol{u}\}) / E_{\mathcal{S}_{i}} .
\end{gathered}
$$

The values of the weights $\omega$ and normalization "energies" $E$ are found with the help of a trial displacement field, $\boldsymbol{v}(\boldsymbol{x})$ in the form of a pure shear wave

$$
\boldsymbol{v}(\boldsymbol{x})=\sin (2 \pi \boldsymbol{k} \cdot \boldsymbol{x})
$$

with displacement orthogonal to the wavevector $\boldsymbol{k}$. The latter is oriented along the largest dimension in the considered region of interest, and defined so that a few wave periods are captured (for a proper statistical sampling of $E_{c}$, and yet no mesh size dependence in $E_{m}$ or $E_{\mathcal{S}_{i}}$ ). The proposed trial field has the benefit of providing an isochoric deformation field. Any other choice would involve a dependence with the Poisson's ratio. 
The normalizing energies are set so that $\hat{\Phi}_{c}(\boldsymbol{v})=1$, hence

$$
\begin{aligned}
E_{c} & =\Phi_{c}(\{\boldsymbol{v}\})=\{\boldsymbol{v}\}^{\boldsymbol{\top}}\left[\boldsymbol{M}^{c}\right]\{\boldsymbol{v}\} \\
E_{m} & =\Phi_{m}(\{\boldsymbol{v}\}) \\
E_{\mathcal{S}_{i}} & =\Phi_{\mathcal{S}_{i}}(\{\boldsymbol{v}\})
\end{aligned}
$$

where $\{\boldsymbol{v}\}$ collects the nodal displacements associated with the trial displacement field $\boldsymbol{v}$. It should be noted that while $E_{c}$ is wavelength independent, the wavelength dependence of $E_{m}$ and $E_{\mathcal{S}_{i}}$ are of fourth order, as identified by dimensional analysis (see Appendix A ). The last two terms work as low-pass filters that locally dampen out abrupt displacement gradients and are tuned by means of characteristic lengths $\xi$. Moreover, since the normalization comes from the global energy, and not the maximum density, the wavevector does not play any role once the image texture is well sampled.

These characteristic lengths $\xi$ are introduced as different parameters for each of the regularizing terms, so as to provide the corresponding weighting terms

$$
\begin{aligned}
\omega_{c} & =1, \\
\omega_{m} & =\left(\xi_{m}|\boldsymbol{k}|\right)^{4}, \\
\omega_{\mathcal{S}_{i}} & =\left(\xi_{\mathcal{S}_{i}}|\boldsymbol{k}|\right)^{4},
\end{aligned}
$$

and

$$
\omega_{t}=\omega_{c}+\omega_{m}+\sum_{i} \omega_{\mathcal{S}_{i}} .
$$

The higher a regularization length $\xi$, the more weight is put on the associated functional. These weights can be intuitively tuned thanks to the normalization procedure. The regularization lengths $\xi$ have the same units as the characteristic length scale $1 /|\boldsymbol{k}|$ of the trial field $\boldsymbol{v}(\boldsymbol{x})$, which usually is expressed in pixels or voxels. When the supporting FE mesh is built using the same units, for any $\xi$ being smaller than the characteristic element length, the associated functional is naturally "deactivated." Conversely, the regularization will take place for any $\xi$ greater than the characteristic element length. This effect can be seen as a filter of size $\xi$ that locally dampens out high spatial frequencies. 


\subsection{Implementation}

The minimization of $\Phi_{t}$ follows the same procedure as the minimization of $\Phi_{c}$. The incremental solution is given by the new linear system

$$
\left(\left[\boldsymbol{M}^{c}\right]+\left[\boldsymbol{M}^{\mathrm{reg}}\right]\right)\{\delta \boldsymbol{u}\}=\{\boldsymbol{b}\}-\left[\boldsymbol{M}^{\mathrm{reg}}\right]\{\boldsymbol{u}\},
$$

with the regularization matrices

$$
\left[\boldsymbol{M}^{\mathrm{reg}}\right]=\left[\boldsymbol{M}^{m}\right]+\sum_{i}\left[\boldsymbol{M}^{\mathcal{S}_{i}}\right]
$$

and

$$
\begin{aligned}
{\left[\boldsymbol{M}^{m}\right] } & =\omega_{m} \frac{E_{c}}{E_{m}}\left[\boldsymbol{K}_{m}\right]^{\top}\left[\boldsymbol{K}_{m}\right], \\
{\left[\boldsymbol{M}^{\mathcal{S}_{i}}\right] } & =\omega_{\mathcal{S}_{i}} \frac{E_{c}}{E_{\mathcal{S}_{i}}}\left[\boldsymbol{K}_{\mathcal{S}_{i}}\right]^{\boldsymbol{\top}}[\boldsymbol{L}]\left[\boldsymbol{K}_{\mathcal{S}_{i}}\right] .
\end{aligned}
$$

The regularized DVC code was implemented within the Correli 3.0 framework [35]. The DVC terms (correlation matrix $\left[\boldsymbol{M}^{c}\right]$ and nodal residual vector $\{\boldsymbol{b}\})$ are computed using (compiled) $\mathrm{C}++$ kernels. These are called into the MATLAB environment so as to iteratively solve equation (29).

\section{Numerical Test Cases}

The goal of these numerical test cases is to observe the impact of the mechanical regularization on a surface and the benefits of using an unstructured mesh.

\subsection{Setup of Test Cases}

First, a numerical material with a rich texture and contrast is created. The sample has "voids" of random sizes and randomly placed in the volume. The size of the created volume is $L_{x} \times L_{y} \times L_{z}$, with $L_{x}=75 \mathrm{vx}, L_{y}=35 \mathrm{vx}$ and $L_{z}=25 \mathrm{vx}$ ( $\mathrm{vx}$ as in voxel). This virtual sample is considered as the reference image in the following analyses. A $3 \mathrm{D}$ rendering is shown in figure 1. 


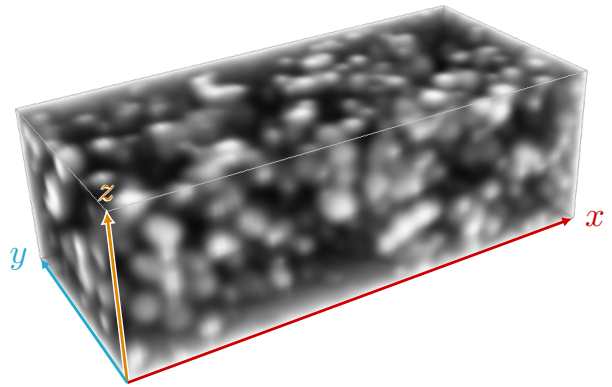

Figure 1: Reference (synthetic) image used in the numerical test cases

Second, two types of meshes are used, namely, a structured and an unstructured mesh. Both meshes are built using tetrahedral elements of length $\ell=5 \mathrm{vx}$ and encompass the same ROI. The structured (resp. unstructured) mesh has 3150 (resp. 2626) elements. Then, a surface (with normal aligned with the $y$-axis and placed at the origin) is selected for the regularization analysis. This surface of interest will be referred to as $\mathcal{S}_{y}$, and is highlighted in figure 2.

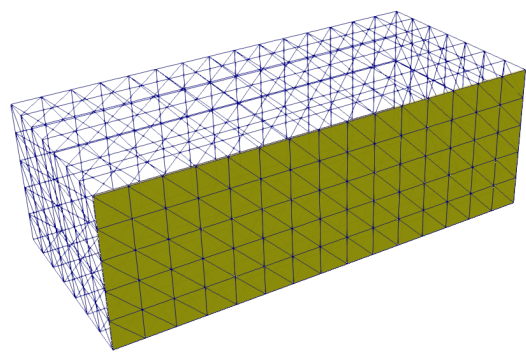

(a)

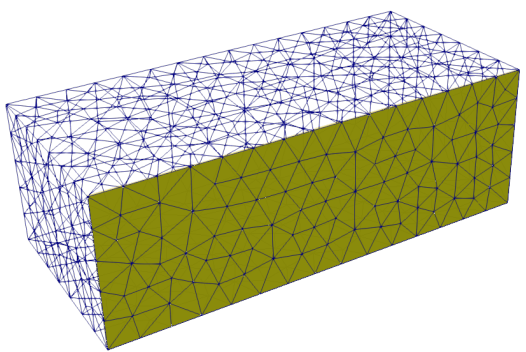

(b)

Figure 2: Structured (a) and unstructured (b) meshes composed of tetrahedral elements. The surface of interest is highlighted

Third, two types of displacement fields are considered for the analysis. The first one has a discontinuity (of 1-voxel amplitude) representative of a crack, and the second is sinusoidal (of 1-voxel amplitude). Using $\hat{\imath}$ as the unit vector along the $x$-direction, the crack-like displacement field is defined as

$$
\boldsymbol{u}_{\text {crack }}^{\mathrm{ref}}(\boldsymbol{x})=0.5 \cdot \operatorname{sign}\left(\boldsymbol{x} \cdot \hat{\boldsymbol{\imath}}-L_{x} / 2\right) \cdot \hat{\boldsymbol{\imath}},
$$


so that a crack appears at the center of the ROI and each half of the specimen moves outward. The sinusoidal displacement is defined via an elastic simulation of an isotropic material with the following boundary conditions applied on the surface $\mathcal{S}_{y}$

$$
\boldsymbol{u}_{\text {sine }}^{\mathrm{ref}}(\boldsymbol{x})=0.5 \cdot \cos \left(2 \pi \cdot \boldsymbol{x} \cdot \hat{\boldsymbol{\imath}} / L_{x}\right) \cdot \hat{\boldsymbol{\jmath}} \quad \text { for } \boldsymbol{x} \in \mathcal{S}_{y},
$$

where $\hat{\jmath}$ is the unit vector along the $y$-direction. For each field, its relevant scalar component is shown in figure 3 .

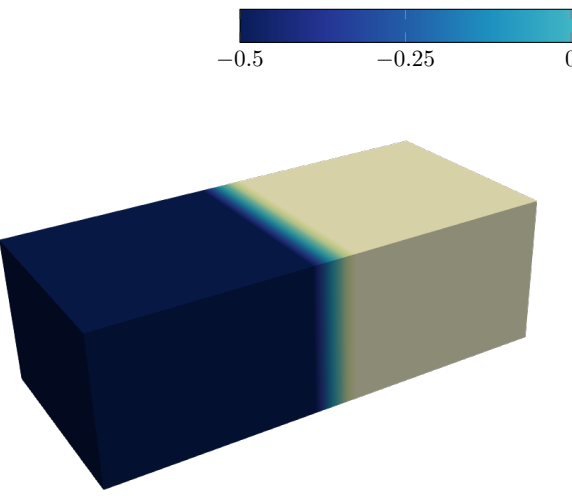

(a) Crack type (along $x$ )

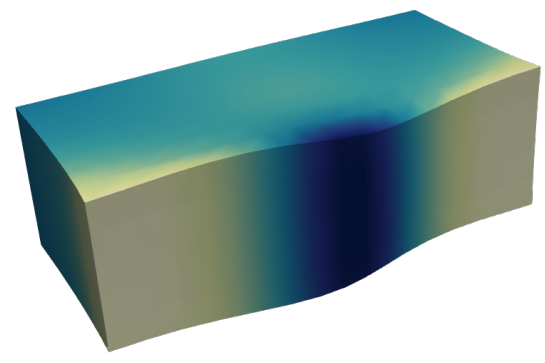

(b) Sine type (along $y$ )

Figure 3: Displacement fields (expressed in voxel) used for the test case, which is magnified for visualization purposes. The directions refer to those shown in figure 1

Once the displacement fields are determined (i.e., they are chosen on the surface and computed in the bulk using a homogeneous linear elastic model), they are employed for generating the virtually deformed images. This is achieved by linearly interpolating the reference image at voxel (pixel) coordinates advected by the appropriate displacement (as given by the fields). Obtaining such appropriate displacements involves transforming the fields expressed in an Lagrangian frame to an Eulerian one (i.e., performing a pull-back of coordinates), which, for case of small displacement gradients, are identical. 


\subsection{Analysis of the Results}

Each of the four available cases are analyzed using different regularization lengths $\xi_{\mathcal{S}_{y}}$ for the surface of interest. These values are chosen with respect to the element length, namely, five values are sampled between $0.2 \ell$ and $4 \ell$. No other regularization was included (bulk or free-surfaces).

The computed displacement fields $\boldsymbol{u}^{\text {cor }}$ are compared to the reference ones. The relevant components are extracted for the nodes belonging to the surface $\mathcal{S}_{y}$ and averaged along the $z$ dimension

$$
U_{\text {crack }}(x)=\frac{1}{L_{z}} \sum\left(\boldsymbol{u}_{\mathrm{crack}}^{\mathrm{cor}}(\boldsymbol{x}) \cdot \hat{\boldsymbol{\imath}}\right),
$$

and

$$
U_{\text {sine }}(x)=\frac{1}{L_{z}} \sum\left(\boldsymbol{u}_{\text {sine }}^{\text {cor }}(\boldsymbol{x}) \cdot \hat{\jmath}\right) \quad \text { for } \boldsymbol{x} \in \mathcal{S}_{y} .
$$

They are shown in figure 4 with the notations $U^{\text {struct }}$ and $U^{\text {unstruct }}$ for the structured and unstructured meshes, respectively. Additionally, since for regularization lengths $\xi_{\mathcal{S}_{y}}<\ell$ the regularization is not activated, all corresponding curves should behave similarly and hide beneath each other. 

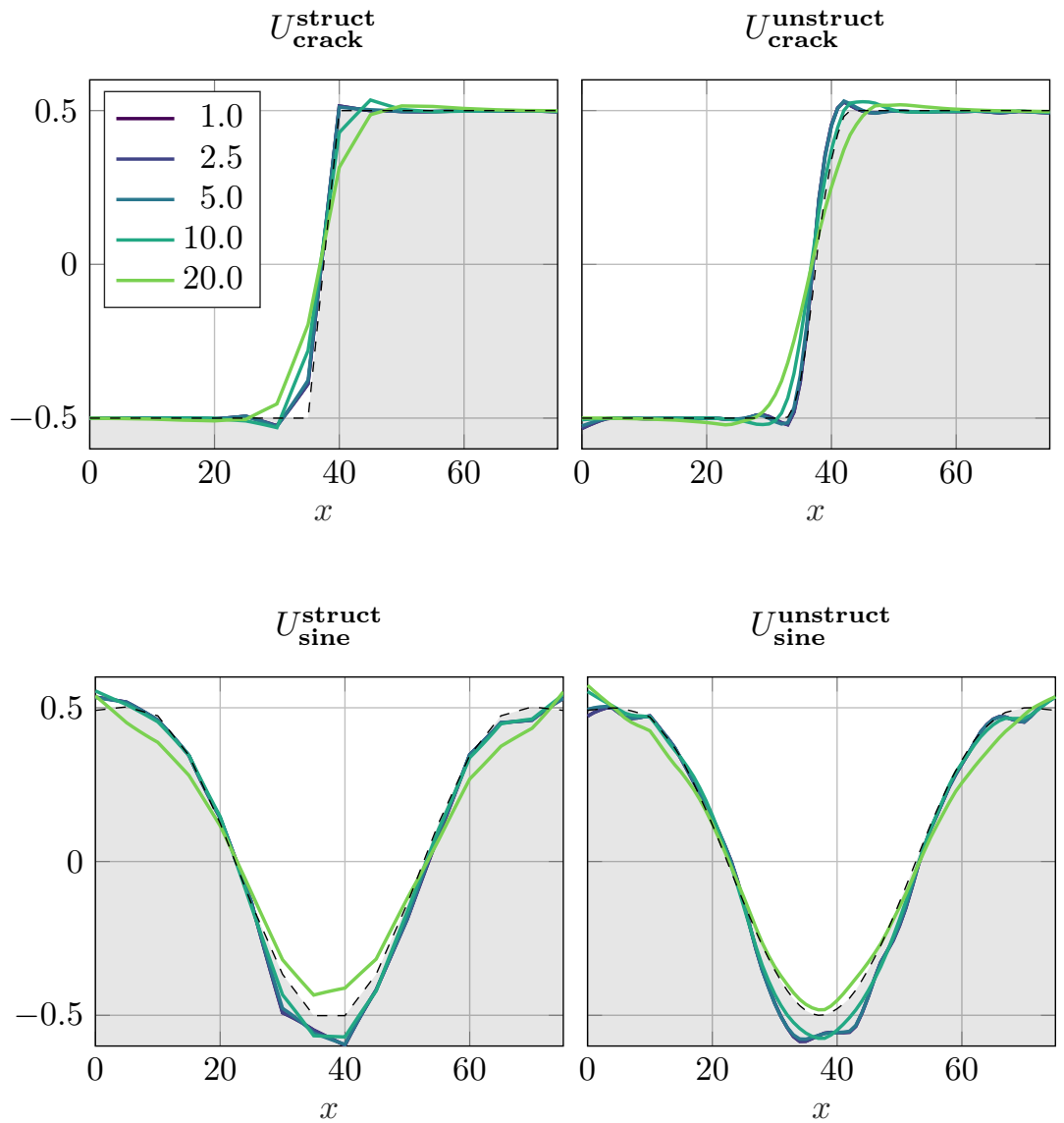

Figure 4: Comparison of reference and averaged displacement fields for all four studied cases. The dashed line shows the reference solution.

First, let us discuss the different displacement types, regardless of the mesh. The crack-like displacement has a discontinuity, that is, it cannot be explained by means of a continuous displacement field locally obeying linear elasticity. Thus, the shorter the regularization length $\xi_{\mathcal{S}_{y}}$ is, the closer $U_{\text {crack }}$ should resemble the step function (i.e., an immediate transition). Conversely, the longer $\xi_{\mathcal{S}_{y}}$ is, the closer $U_{\text {crack }}$ should resemble the sigmoid function (i.e., a slow transition). Similarly, the prescribed displacement field $U_{\text {sine }}$ has a well defined characteristic length ( $L_{x}$ in the present case). As stated before, the regulariza- 
tion scheme works as a low-pass filter with a cut-off frequency defined by the regularization length $\xi_{\mathcal{S}_{y}}$. Hence, when the regularization length $\xi_{\mathcal{S}_{y}}$ is less than the characteristic length of the original sinusoid, regularization should have no effect. Conversely, for larger values, this test case should simply dampen out the amplitude of the prescribed field, and should not alter its frequency and phase. Both of these features are validated in figure 4 for both types of meshes.

Second, let us discuss the effect of both meshes. The unstructured mesh provides a better discretization of the studied fields. That is, in spite of having a lesser number of nodes, their "random" arrangement helps to better capture the considered displacement fields. This is, in spirit, similar to the advantage of random search versus classical search of hyper-parameters for optimization problems [36].

\section{Experimental Case}

The goal of this section is to present the advantages of the technique for a real tensile test performed on a pre-cracked sample made of spheroidal graphite cast iron.

\subsection{Analysis Preparation}

A region of interest of $4.2 \times 1.9 \times 1.6 \mathrm{~mm}$ is imaged using a micro-CT scanner (North Star Imaging X50+) with a resolution of $7 \mu \mathrm{m}$. This sample was cut out of a bigger specimen that was pre-fatigued with a load shedding technique in order to avoid having a large plastic zone around the crack front [37, 38]. The objective of the present test was to analyze ductile tearing in a cracked sample.

Seven tomographic scans were acquired at increasingly loading stages (the load is kept constant while the sample is being scanned). These $3 \mathrm{D}$ reconstructed volumes will be referred to as S0, S1, S2, S3, S4, S5 and S6. A 2D slice of each scan is shown in figure 5 alongside the load at which each scan was acquired. Scans S0, S1 and S2 are obtained in the elastic regime of the sample, thus the crack does not open very much. This observation allows scans S0 and S1 to be 
used for assessing baseline levels and measurement uncertainties. Conversely, crack opening is more pronounced in scans S3 and S4 when the sample has yielded. Last, ductile tearing occurs for scans S5 and S6.

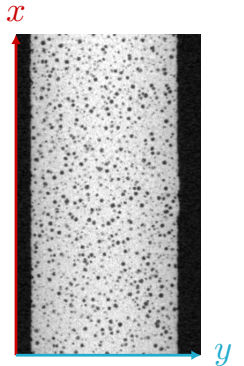

(a) S0, $61 \mathrm{~N}$

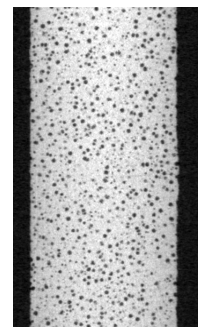

(b) $\mathrm{S} 1,196 \mathrm{~N}$

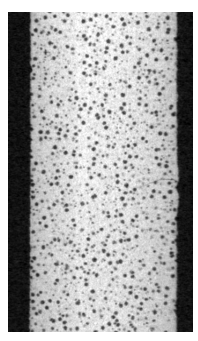

(c) $\mathrm{S} 2,282 \mathrm{~N}$

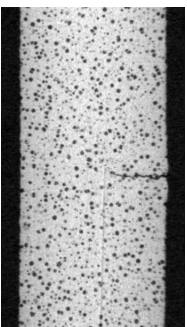

(d) $\mathrm{S} 3,646 \mathrm{~N}$

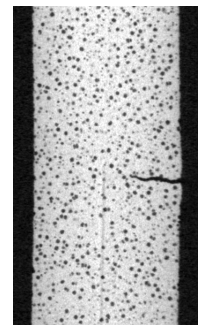

(e) $\mathrm{S} 4,720 \mathrm{~N}$

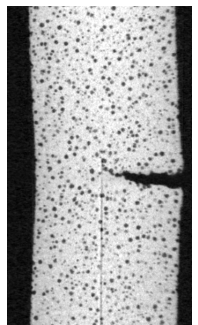

(f) $\mathrm{S} 5,805 \mathrm{~N}$

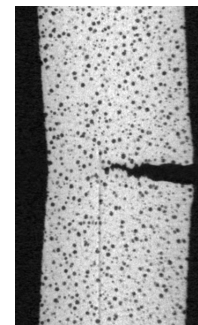

(g) $\mathrm{S} 6,730 \mathrm{~N}$

Figure 5: $(x-y)$ mid-thickness sections for all scans showing the test history (crack opening and tearing) with the respective applied loads

The first scan S0, whose 3D rendering is shown in figure $6 \mathrm{a}$, is chosen as the reference for the correlation procedure. Thus, it is used for constructing the support of the FE mesh. A first mesh that roughly aligns with the image is created, it needs to be adjusted onto the image. The adjustment [39] is based on two binary masks, namely, one created from the reference scan and a second created from the domain of the FE mesh. DVC is performed between both masks [40] with a reduced kinematics. Applying the obtained displacement field to the FE mesh, provides the necessary geometry for the mesher [17]. As such, the obtained unstructured tetrahedral FE mesh fits very precisely to the sample surfaces. It should be noted that, while faintly perceptible, none of the lateral 
surfaces are aligned with the image axes. The mesh is constructed so that the characteristic element length is $\ell=25 \mathrm{vx}$. While it could be considered small (as compared to the material microstructure), it was chosen so that unregularized DVC would encounter difficulties for converging (i.e., $\sqrt[3]{V_{T}} \approx 14 \mathrm{vx}$, where $V_{T}$ the volume of each tetrahedral element).

It is noteworthy that the presence of the crack is not taken into account, and hence regularization will tend to bridge the two crack faces by smearing out the displacement discontinuity over a region controlled by the regularization length (as was observed in the previous test case). While the strategy of introducing a discontinuity in the mesh would have been a better suited option [41, 42], the model "error" of ignoring the kinematic discontinuity allows the role of regularization to be better highlighted.

Moreover, in order to illustrate the versatility of the proposed regularization scheme for Dirichlet boundaries, a more complex geometry of the top boundary is considered. A new "cropped" mesh is created by removing the uppermost layer of elements from the original "complete" FE mesh. This leads to a new mesh with a very uneven (jagged) upper surface. Since the lateral surfaces are traction-free, they are considered of Neumann type. On the contrary, the upper and bottom surfaces cut through the sample, thus they are considered of Dirichlet type. Both meshes and the corresponding Dirichlet surfaces $\mathcal{S}_{x}$ are shown in figure 6 . 


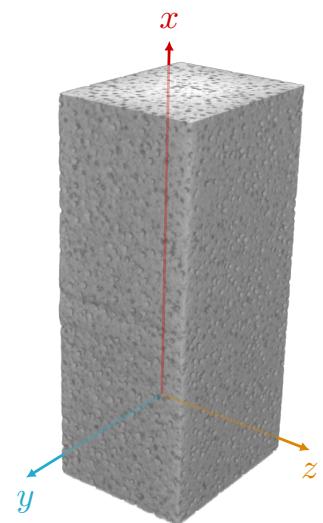

(a)

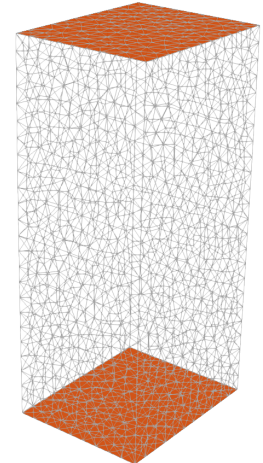

(b)

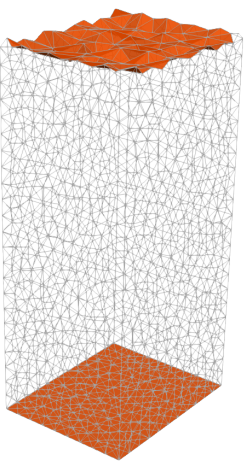

(c)

Figure 6: (a) Volume rendering of the reference scan S0. Visualization of the (b) "complete" and (c) "cropped" meshes with the corresponding Dirichlet surfaces being highlighted

Using both meshes, it is proposed to study three regularization scenarios:

- none: with no regularization

- bulk: using only bulk regularization

- all: using bulk and surface regularization

All three cases are based on global DVC, and two are complemented by the corresponding regularization. For this reason, the so-called "none" case, which corresponds to the standard version of global DVC [43], may already be considered as a "regularized" version of the more common (i.e., local) DVC approaches $[1,2,12,13]$ since displacement continuity is enforced over the whole ROI. Thus, when convergence reveals difficult without regularization, most of the existing local DVC codes would encounter even more obstacles for converging.

DVC is to be carried out between the reference configuration and each one of the remaining six scans (these constitute all the deformed configurations). This set of correlations will be performed in a direct manner, that is, the displacement field found for the previous scan is used as an initialization for the current one 
even though the reference configuration is always the same. Only the first computation (for scan S1) is performed with no initialization (all displacements are set to zero). This procedure will be applied (individually) to each of the three scenarios (none, bulk and all).

To ensure robustness and low uncertainties, the regularization length for each DVC calculation (i.e., for bulk and all cases) is progressively relaxed as the algorithm progresses. This process helps transitioning from an "educated guess" of the displacement field (i.e., heavily regularized) toward a more faithful representation of the actual field (i.e., less regularized). The proposed relaxation procedure consists of performing four successive correlation steps, each with shorter regularization lengths. These lengths are obtained by defining a scale factor of two and the length at the finer scale $\xi$. Then, the correlation steps will employ $8 \xi, 4 \xi, 2 \xi$ and $1 \xi$ (in that order). The transition between such steps is performed either when a maximum of 50 iterations (i.e., 200 maximum per scan) is reached or when convergence is achieved. The convergence criterion for each correlation step is defined with respect to the $L_{2}$ norm of the GaussNewton update $\|\delta \boldsymbol{u}\|<0.01$ voxel. Moreover, the solution of a given correlation step will be used as initialization for the following one. Similar criteria are employed for the non-regularized none case, in which case a single correlation step is performed per scan.

Last, for the sake of simplicity, the regularization lengths are set to $\xi_{m}=\alpha \cdot \ell$ for bulk and Neumann DOFs and $\xi_{\mathcal{S}}=\alpha \cdot \xi_{m}$ for the concerned (Dirichlet) surfaces. This allows setting $\alpha=2$ for the analyses using the "complete" FE mesh and $\alpha=4$ for the analyses using the "cropped" FE mesh. These are two fully separate analyses and are carried out independently from each other. Both parameterizations are tailored so as to ensure final convergence for each case (i.e., the "cropped" case is more complex than the "complete" one). 


\subsection{Results}

Let us analyze the results with the "complete" FE mesh (shown in figure 6b) from the perspective of the correlation residual

$$
\sigma=\frac{1}{\Delta f} \sqrt{\left\langle\eta(\boldsymbol{x})^{2}\right\rangle_{\boldsymbol{x}}}
$$

normalized by the dynamic range $\Delta f=\max (f)-\min (f)$. The residuals $\sigma$ are computed for each of the deformed configurations. These results are reported in table 1 along with the total number of iterations $N_{i t}$ per scan.

Table 1: Number of iterations and final residual for different regularization strategies (converged results are shown in boldface). All strategies are based on global DVC [43]

\begin{tabular}{ccccccccc}
\hline & \multicolumn{2}{c}{ none } & & \multicolumn{2}{c}{ bulk } & & \multicolumn{2}{c}{ all } \\
\cline { 8 - 9 } & $N_{i t}$ & $\sigma$ & & $N_{i t}$ & $\sigma$ & & $N_{i t}$ & $\sigma$ \\
\hline S1 & 200 & $5.02 \%$ & & $\mathbf{9 4}$ & $\mathbf{3 . 2 4 \%}$ & & $\mathbf{1 3}$ & $\mathbf{3 . 2 4 \%}$ \\
S2 & 200 & $10.15 \%$ & & $\mathbf{1 7 6}$ & $\mathbf{3 . 2 6 \%}$ & & $\mathbf{1 8}$ & $\mathbf{3 . 2 5 \%}$ \\
S3 & 200 & $11.23 \%$ & & 200 & $5.09 \%$ & & $\mathbf{1 6}$ & $\mathbf{4 . 4 4 \%}$ \\
S4 & 200 & $11.38 \%$ & & $\mathbf{1 9 6}$ & $\mathbf{3 . 5 4 \%}$ & & $\mathbf{1 4}$ & $\mathbf{3 . 4 9 \%}$ \\
S5 & 200 & $11.29 \%$ & & 200 & $4.13 \%$ & & $\mathbf{3 3}$ & $\mathbf{4 . 1 1 \%}$ \\
S6 & 200 & $11.03 \%$ & & 200 & $5.68 \%$ & & $\mathbf{6 5}$ & $\mathbf{5 . 3 7 \%}$ \\
\hline
\end{tabular}

With regard to the baseline measurement (between S0 and S1), the none code does not converge (by design). Conversely, bulk and all regularizations lead to convergence with $\sigma=3.24 \%$, a low value when dealing with tomographic data. For the remaining scans, it should be noted that all computations converged for the all case. Yet only two and zero calculations converged for the bulk and none regularizations, respectively. Even in bulk cases that converged (i.e., S2, S4), it required a significantly higher number of iterations than in the all case. This observation shows the gain associated with surface regularization. 
It is worth mentioning that the load increment between scans is not constant. As can be seen in figure 5, the load increase between scans S2 and S3 (364 N) is much more important than between scans S3 and S4 $(74 \mathrm{~N})$. Additionally, the registration is always performed with respect to the reference scan S0, and the correlation procedures are all initialized with the results from the previous one. Hence, the correlation step for scan S3 is much more complicated than for scan S4. This leads to a particular case of convergence for the bulk case, in which the scan S4 converges even though S3 did not.

A more detailed analysis of the correlation residual $\sigma$ and the measured displacement fields $\boldsymbol{u}(\boldsymbol{x})$ allow the benefits of the proposed surface regularization to be commented. The former is shown in figure 7, with the residuals $\sigma(x)$ as functions of the $x$ coordinate for each of the scans and both regularization cases (i.e., bulk and all). The latter are reported in figure 8 for the last scan S6 and both regularization cases by comparing the measured 3D displacements with the $3 \mathrm{D}$ rendering of the scan. These results provide a clear insight into the beneficial effect of the proposed surface regularization, namely, the all case easily handles the top-most surface whereas correlations with no surface regularization (i.e., none and bulk) cannot. It should be noted that, even though the registration was successful in a significant part of the sample using the bulk regularization, overall convergence was not achieved. However, just by taking this surface into consideration (i.e., all), the problem is easily solved. 


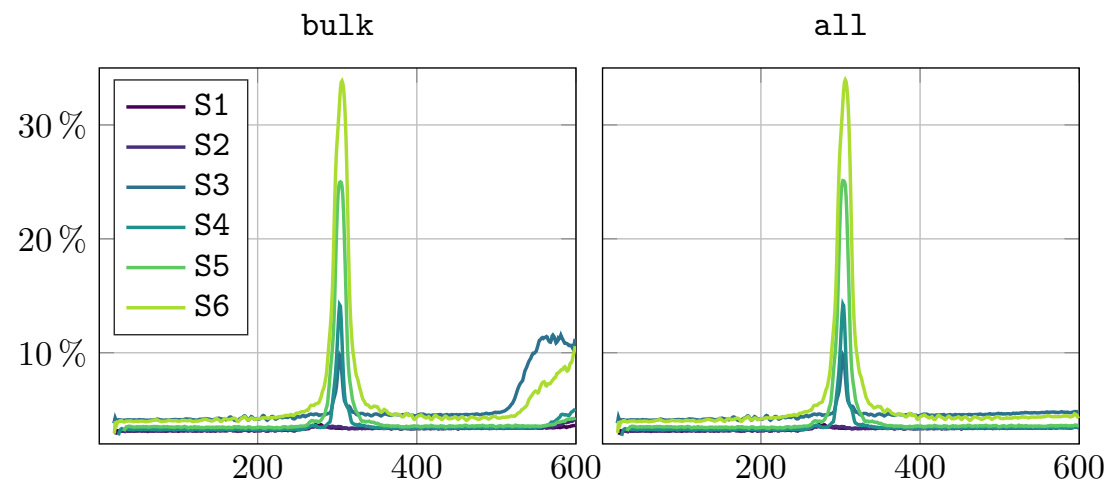

Figure 7: Residuals averaged over $(y-z)$ cross-sections as a function of $x$ for the two regularization cases. The peaks correspond to the crack.

As a side remark, a slight increase of residuals between scans can be seen in figure 7. This effect is due to ductile tearing, namely, crack opening and propagation introduces many "black" (i.e., zero valued) voxels in the ROI of the deformed configuration. Because the residuals are rescaled globally, they increase alongside the cracking mechanisms. 


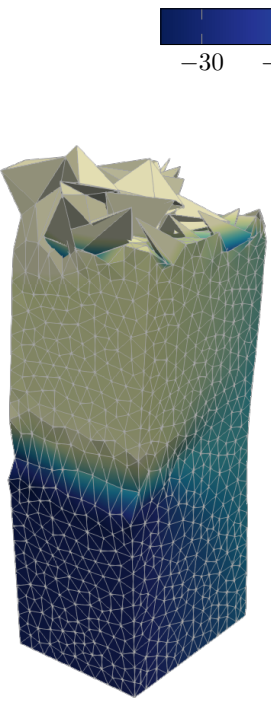

(a) bulk

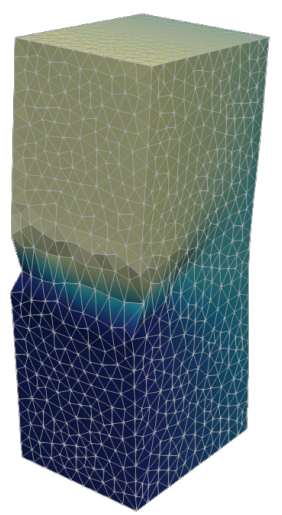

(b) all

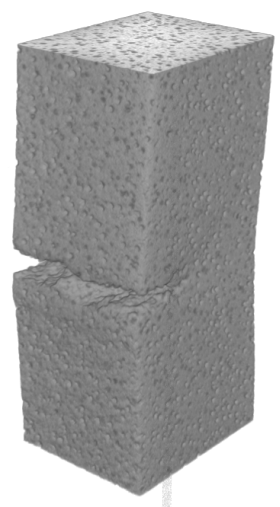

(c) $\mathrm{S} 6$

Figure 8: Longitudinal displacement (expressed in voxels) for the cases with (a) bulk and (b) all regularizations using the "complete" FE mesh. (c) 3D rendering of scan $\mathrm{S} 6$

Last, figure 9 shows the mid-thickness slice of each residual $\eta(\boldsymbol{x})$ at convergence when all DOFs are regularized. For scan S2, the crack does not open sufficiently to have a signature in the gray level residuals. Conversely, the crack opens more significantly for scans S3 and S4 but does not propagate. Ductile tearing occurs in scans S5 and S6. Apart from the zones around the crack surface, the residuals are identical in levels as in the baseline scan S1. Moreover, when using the "cropped" mesh neither the none or the bulk regularization scenarios converge. Convergence is achieved only when the complete regularization is used for the Dirichlet surfaces (i.e., all). The residuals corresponding to the "cropped" mesh are virtually identical to those obtained using the "complete" mesh (e.g., figures $9 \mathrm{f}$ and $9 \mathrm{l}$ ) even though the uppermost surface is very rough. All these observations further validate the regularization strategy for arbitrarily complex surface geometries. 


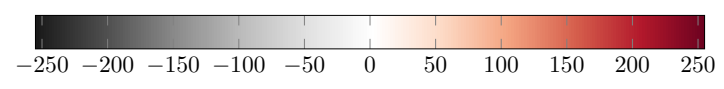

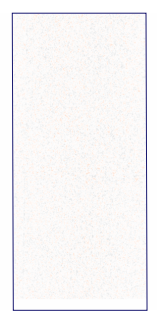

(a) S1

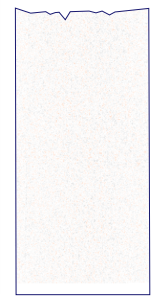

(g) S1

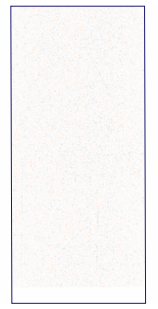

(b) S2

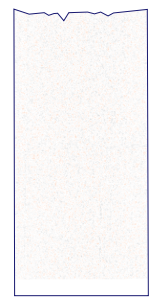

(h) S2

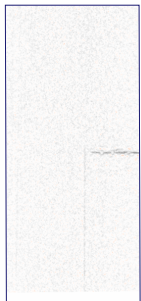

(c) $\mathrm{S} 3$

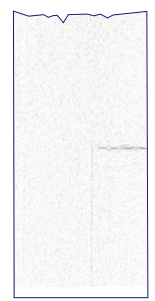

(i) $\mathrm{S} 3$

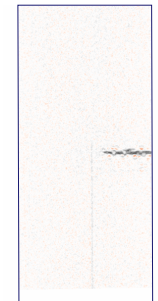

(d) $\mathrm{S} 4$

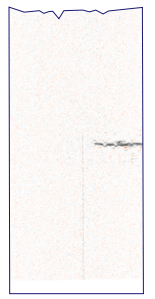

(j) $\mathrm{S} 4$

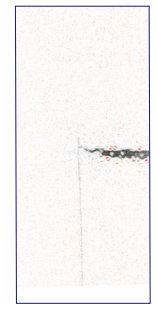

(e) S5

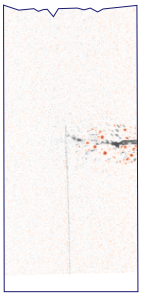

(k) S5

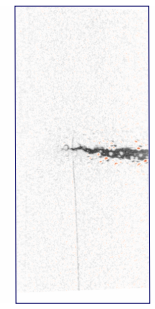

(f) $\mathrm{S} 6$

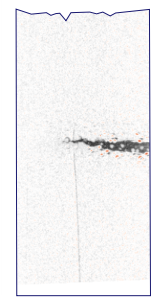

(l) $\mathrm{S} 6$

Figure 9: $(x-y)$ mid-thickness gray level residuals with all DOFs regularized, using (a)-(f) the "complete" mesh or (g)-(l) the "cropped" mesh. The outline indicates the mesh boundary

\section{Conclusions}

The complete mechanical regularization proposed herein opens many possibilities in the field of global (i.e., FE-based) Digital Image and Volume Correlation. First, the method is directly applicable to both 3D and 2D cases (i.e., DVC and DIC) with no need for developing customized regularization operators. Likewise, the use of the Laplacian operator allows for the study of arbitrarily complex shapes, that is, not limited to flat (resp. straight) surfaces (resp. boundaries) [20, 22, 23]. This observation implies that objects can now be studied in their entirety, instead of traditional approaches that limit their regions of interest to the interior of the object (i.e., the bulk). Furthermore, 
each of these boundaries can be accounted for differently, according to the initial conditions (i.e., Dirichlet or Neumann type).

Since the technique is an extension to the bulk mechanical regularization, it naturally inherits all its benefits. As such, it provides a mechanically admissible displacement field that best registers the images. Additionally, it provides lower levels of uncertainty, even when dealing with images of poor quality. It further reveals the advantages of regularization over some traditional multiscale approaches, in particular for unstructured meshes.

The results presented herein (numerical and real tests) confirm all these properties. A noteworthy benefit of the complete mechanical regularization is its fast convergence. In practical terms, this result implies that even complex cases can be treated both robustly and fast. Moreover, besides the definition of the FE mesh, the only parameters required for the proposed regularization scheme are the (very intuitive) regularization lengths for the bulk and boundaries. Likewise, simple cases, which may not "require" regularization, can benefit from faster convergence. This desirable behavior (i.e., well-conditioning) is shown in the analyzed experiment where a complex case was swiftly solved (i.e., low number of iterations) using the complete regularization scheme.

The regularization used herein was based on plain linear elasticity. If a better appreciation of the sample constitutive law is available, the corresponding regularization can be generalized. This would still penalize the unbalanced forces and allow larger regularization lengths to be used.

Last, while most of the discussion focused on 3D aspects (i.e., DVC), it is important to stress that it is also valid for $2 \mathrm{D}$ analyses (via DIC).

\section{Acknowledgements}

The authors are very grateful to Prof. J.-Y. Buffière and Dr. N. Limodin for kindly providing and preparing the test sample. They also wish to thank Dr. A. Bouterf and Mr. B. Smaniotto for performing the test reported herein. This work was supported by the PRC MECACOMP, project co-funded by 
DGAC and Safran group, involving Safran group companies, ONERA, CNRS and other academic partners. This work has also benefited from the support of the French "Agence Nationale de la Recherche" through the "Investissements d'avenir" program under the reference "ANR-10-EQPX-37 MATMECA".

[1] H. Schreier, J.-J. Orteu, M. A. Sutton, Image Correlation for Shape, Motion and Deformation Measurements, Springer US, ISBN 978-0-387-78746-6, 10.1007/978-0-387-78747-3, 2009.

[2] M. A. Sutton, Computer Vision-Based, Noncontacting Deformation Measurements in Mechanics: A Generational Transformation, Applied Mechanics Reviews 65 (5) (2013) 050000, ISSN 0003-6900, 10.1115/1.4024984.

[3] M. Bertero, T. A. Poggio, V. Torre, Ill-Posed Problems in Early Vision, Proceedings of the IEEE 76 (8) (1988) 869-889, ISSN 15582256, 10.1109/ 5.5962 .

[4] F. Hild, S. Roux, Digital Image Correlation, in: P. Rastogi, E. Hack (Eds.), Optical Methods for Solid Mechanics. A Full-Field Approach, chap. 5, Wiley-VCH, Weinheim (Germany), 183-228, 2012.

[5] M. Bornert, F. Brémand, P. Doumalin, J. C. Dupré, M. Fazzini, M. Grédiac, F. Hild, S. Mistou, J. Molimard, J. J. Orteu, L. Robert, Y. Surrel, P. Vacher, B. Wattrisse, Assessment of digital image correlation measurement errors: Methodology and results, Experimental Mechanics 49 (3) (2009) 353-370, ISSN 00144851, 10.1007/s11340-008-9204-7.

[6] H. Leclerc, J.-N. Périé, F. Hild, S. Roux, Digital volume correlation: what are the limits to the spatial resolution?, Mechanics \& Industry 13 (6) (2012) 361-371, ISSN 2257-7777, 10.1051/meca/2012025.

[7] G. Broggiato, Adaptive image correlation technique for full-field strain measurement, in: C. Pappalettere (Ed.), 12th Int. Conf. Exp. Mech., McGraw Hill, Lilan (Italy), 20-421, 2004. 
[8] Y. Sun, C. K. Wong, F. Su, J. H. L. Pang, Finite element formulation for a digital image correlation method, Applied Optics 44 (34) (2005) 7357-7363, ISSN 0003-6935, 10.1364/AO.44.007357.

[9] G. Besnard, F. Hild, S. Roux, Finite-element displacement fields analysis from digital images: Application to Portevin-Le Châtelier bands, Experimental Mechanics 46 (6) (2006) 789-803, ISSN 00144851, 10.1007/ s11340-006-9824-8.

[10] A. Baldi, F. Bertolino, A Meshless Global DIC Approach, in: Advancement of Optical Methods in Experimental Mechanics, vol. 3, Springer, ISBN 9783-319-22445-9, 175-180, 10.1007/978-3-319-22446-6, 2016.

[11] S. Roux, F. Hild, P. Viot, D. Bernard, Three-dimensional image correlation from X-ray computed tomography of solid foam, Composites Part A: Applied Science and Manufacturing 39 (8) (2008) 1253-1265, ISSN 1359835X, 10.1016/j.compositesa.2007.11.011.

[12] B. K. Bay, T. S. Smith, D. P. Fyhrie, M. Saad, Digital volume correlation: Three-dimensional strain mapping using X-ray tomography, Experimental Mechanics 39 (3) (1999) 217-226, ISSN 0014-4851, 10.1007/BF02323555.

[13] B. K. Bay, Methods and applications of digital volume correlation, The Journal of Strain Analysis for Engineering Design 43 (8) (2008) 745-760, ISSN 0309-3247, 10.1243/03093247JSA436.

[14] S. Avril, M. Bonnet, A. S. Bretelle, M. Grédiac, F. Hild, P. Ienny, F. Latourte, D. Lemosse, S. Pagano, E. Pagnacco, F. Pierron, Overview of identification methods of mechanical parameters based on full-field measurements, Experimental Mechanics 48 (4) (2008) 381-402, 10.1007/ s11340-008-9148-y.

[15] M. Shakoor, A. Buljac, J. Neggers, F. Hild, T. F. Morgeneyer, L. Helfen, M. Bernacki, P. O. Bouchard, On the choice of boundary conditions for 
micromechanical simulations based on 3D imaging, International Journal of Solids and Structures 112 (2017) 83-96, 10.1016/j.ijsolstr.2017.02.018.

[16] F. Hild, S. Roux, Comparison of local and global approaches to digital image correlation, Experimental Mechanics 52 (9) (2012) 1503-1519.

[17] C. Geuzaine, J. F. Remacle, Gmsh: A 3-D finite element mesh generator with built-in pre- and post-processing facilities, International Journal for Numerical Methods in Engineering 79 (11) (2009) 1309-1331, ISSN 00295981, 10.1002/nme.2579.

[18] A. N. Tikhonov, V. Y. Arsenin, Solutions of ill-posed problems, Winston, 1977.

[19] J. Réthoré, S. Roux, F. Hild, An extended and integrated digital image correlation technique applied to the analysis of fractured samples, European Journal of Computational Mechanics 18 (3-4) (2009) 285-306, ISSN 17797179, 10.3166/ejcm.18.285-306.

[20] Z. Tomicevic, F. Hild, S. Roux, Mechanics-Aided Digital Image Correlation, The Journal of Strain Analysis for Engineering Design 48 (5) (2013) 330343.

[21] D. Claire, F. Hild, S. Roux, A finite element formulation to identify damage fields: the equilibrium gap method, International Journal for Numerical Methods in Engineering 61 (2) (2004) 189-208.

[22] H. Leclerc, J. N. Périé, S. Roux, F. Hild, Voxel-Scale Digital Volume Correlation, Experimental Mechanics 51 (4) (2011) 479-490, ISSN 00144851, 10.1007/s11340-010-9407-6.

[23] T. Taillandier-Thomas, S. Roux, T. F. Morgeneyer, F. Hild, Localized strain field measurement on laminography data with mechanical regularization, Nuclear Instruments and Methods in Physics Research, Section B: Beam Interactions with Materials and Atoms 324 (2014) 70-79, ISSN 0168583X, 10.1016/j.nimb.2013.09.033. 
[24] S. Timoshenko, J. Goodier, Theory of elasticity, Mcgraw Hill, 1951.

[25] O. C. Zienkiewicz, The finite element method, vol. 3, McGraw-hill London, 1977.

[26] H. A. Bruck, S. R. McNeill, M. A. Sutton, W. H. Peters, Digital image correlation using Newton-Raphson method of partial differential correction, Experimental mechanics 29 (3) (1989) 261-267.

[27] E. Süli, D. F. Mayers, An introduction to numerical analysis, Cambridge university press, 2003.

[28] B. K. Horn, B. G. Schunck, Determining optical flow, Artificial Intelligence 17 (1-3) (1981) 185-203, ISSN 00043702, 10.1016/0004-3702(81)90024-2.

[29] B. Scher, J. Modersitzki, Fast diffusion registration, Contemporary Mathematics 313 (2002) 117-128.

[30] J.-C. Passieux, J.-N. Périé, High resolution digital image correlation using proper generalized decomposition: PGD-DIC, International Journal for Numerical Methods in Engineering 92 (6) (2012) 531-550, ISSN 00295981, 10.1002/nme.4349.

[31] T. J. Hughes, The finite element method: linear static and dynamic finite element analysis, Courier Corporation, 2012.

[32] M. Reuter, F.-E. Wolter, N. Peinecke, Laplace-spectra as fingerprints for shape matching, in: SPM '05 Proceedings of the 2005 ACM symposium on Solid and physical modeling, ISBN 1595930159, ISSN 18117783, 101-106, 10.1145/1060244.1060256, 2005.

[33] M. Reuter, F. E. Wolter, N. Peinecke, Laplace-Beltrami spectra as 'ShapeDNA' of surfaces and solids, CAD Computer Aided Design 38 (4) (2006) 342-366, ISSN 00104485, 10.1016/j.cad.2005.10.011. 
[34] M. Reuter, S. Biasotti, D. Giorgi, G. Patanè, M. Spagnuolo, Discrete Laplace-Beltrami operators for shape analysis and segmentation, Computers and Graphics (Pergamon) 33 (3) (2009) 381-390, ISSN 00978493, 10.1016/j.cag.2009.03.005.

[35] H. Leclerc, J. Neggers, F. Mathieu, F. Hild, S. Roux, Correli 3.0, IDDN. FR.001.520008.000.S.P.2015.000.31500, 2015.

[36] J. Bergstra, Y. Bengio, Random Search for Hyper-Parameter Optimization, Journal of Machine Learning Research 13 (Feb) (2012) 281-305, ISSN 1532$4435,10.1162 / 153244303322533223$.

[37] N. Limodin, J. Réthoré, J. Y. Buffière, A. Gravouil, F. Hild, S. Roux, Crack closure and stress intensity factor measurements in nodular graphite cast iron using three-dimensional correlation of laboratory X-ray microtomography images, Acta Materialia 57 (14) (2009) 4090-4101, ISSN 13596454, 10.1016/j.actamat.2009.05.005.

[38] N. Limodin, J. Réthoré, J. Y. Buffière, F. Hild, S. Roux, W. Ludwig, J. Rannou, A. Gravouil, Influence of closure on the 3D propagation of fatigue cracks in a nodular cast iron investigated by X-ray tomography and 3D volume correlation, Acta Materialia 58 (8) (2010) 2957-2967, ISSN 13596454, 10.1016/j.actamat.2010.01.024.

[39] R. Gras, H. Leclerc, F. Hild, S. Roux, J. Schneider, Identification of a set of macroscopic elastic parameters in a $3 \mathrm{D}$ woven composite: Uncertainty analysis and regularization, International Journal of Solids and Structures 55 (2015) 2-16, ISSN 00207683, 10.1016/j.ijsolstr.2013.12.023.

[40] J. Réthoré, M. François, Curve and boundaries measurement using Bsplines and virtual images, Optics and Lasers in Engineering 52 (2014) $145-155$.

[41] S. Roux, F. Hild, H. Leclerc, Mechanical assistance to DIC, Procedia IUTAM 4 (2012) 159-168, ISSN 22109838, 10.1016/j.piutam.2012.05.018. 
[42] E. Fagerholt, T. Borvik, O. S. Hopperstad, Measuring discontinuous displacement fields in cracked specimens using digital image correlation with mesh adaptation and crack-path optimization, Optics and Lasers in Engineering 51 (3) (2013) 299-310, ISSN 01438166, 10.1016/j.optlaseng.2012. 09.010 .

[43] S. Roux, F. Hild, Digital image mechanical identification (DIMI), Experimental Mechanics 48 (4) (2008) 495-508.

\section{Appendix A. Scaling of regularization length}

To illustrate the scaling of the boundary term, let us consider a $2 \mathrm{D}$ case $(x, y)$ where a simple solution is available. The domain to be considered is a semi-infinite plane $x<0$. Then, considering an antiplane situation (i.e., $u_{x}=u_{y}=0$ ), the displacement in the direction perpendicular to the plane is defined as (with the real part omitted)

$$
u_{z}(x, y)=\exp (q \cdot x+i \cdot q \cdot y)
$$

for any real $q$. It is observed that, since

$$
\begin{aligned}
& u_{z, x x}=q^{2} u_{z} \\
& u_{z, y y}=-q^{2} u_{z},
\end{aligned}
$$

then $u_{z}$ is the solution to a homogeneous elastic problem as it obeys $\nabla^{2} u_{z}=0$. From the antiplane condition, the only non-vanishing strain components are

$$
\begin{aligned}
& \epsilon_{x z}(x, y)=q \cdot u_{z} \\
& \epsilon_{y z}(x, y)=i \cdot q \cdot u_{z}
\end{aligned}
$$

and the corresponding symmetric component $\epsilon_{z x}$ and $\epsilon_{z y}$. Thus, the stress tensor reduces to

$$
\boldsymbol{\sigma}=2 \mu \cdot \boldsymbol{\epsilon},
$$


and the normal traction along the boundary $x=0$ reads

$$
\begin{aligned}
t_{z}(y) & =2 \mu \cdot \epsilon_{x z}(0, y), \\
& =2 \mu \cdot q \cdot \exp (i \cdot q \cdot y) .
\end{aligned}
$$

Last, the proposed regularization is based on the quadratic norm of the gradient of $t_{z}$ along $y$ (including real part that was earlier omitted)

$$
\begin{aligned}
r & =\left|\partial_{y} t_{z}(y)\right|^{2} \\
& =\left|2 \mu \cdot q^{2} \cdot \sin (q \cdot y)\right|^{2},
\end{aligned}
$$

which, when integrated over $y$ along a large distance (as compared to $1 / q$ ), becomes

$$
\int_{0}^{L_{y}} r \mathrm{~d} y \approx 2 \mu^{2} \cdot q^{4} \cdot L_{y}
$$

Hence, the scaling of the regularization kernel is $q^{4}$, the fourth power also observed in bulk regularization, where $q=2 \pi|\boldsymbol{k}|$ in equation (25). 